\title{
Gastrointestinal lymphoid pseudotumoral hyperplasia: report of four pediatric cases
}

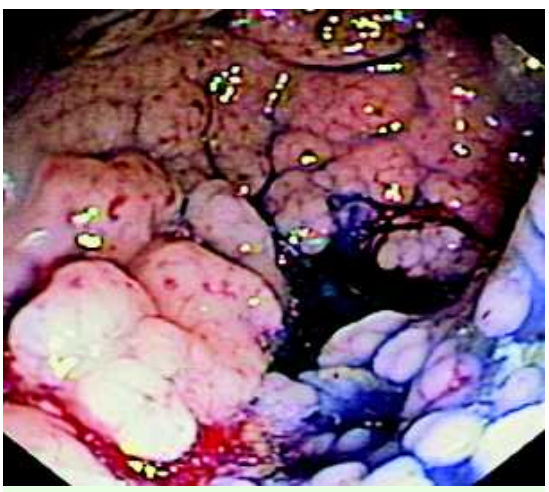

Fig. 1 Endoscopy aspect of lymphoid nodular hyperplasia in the left colon after chromoendoscopy using indigo carmine dye.

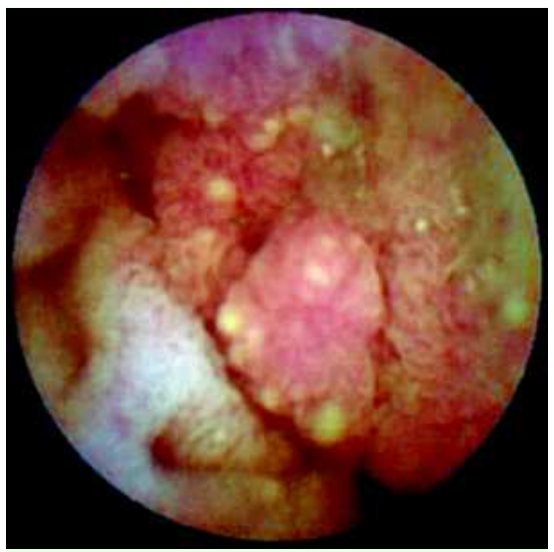

Fig. 2 Video capsule appearance of the jejunal lesions.

We report on four pediatric patients (๑ Table 1, Fig.1-3) with lymphoid pseudotumoral hyperplasia (LPH), two with an atypical localization (diffuse disease). The lesion diameters varied from 7 to $30 \mathrm{~mm}$.

In our patients, the treatment was: conservative (patient 1 ), surgery (patient 2 ), corticosteroids (patient 3 ), and a milkfree diet (patient 4). Complete remission of lesions and/or clinical improvement were seen in all cases. In the three clinically managed cases, there were no changes during the follow-up ( 6 years for patient 2, 4 years for patient 3 , and 6 months for patient 4 ).

Lymphoid nodular hyperplasia ( $\mathrm{LNH}$ ) is a common, nonpathologic finding in children [1]. The ileum and colon are commonly affected $[1,2]$. An atypical form is

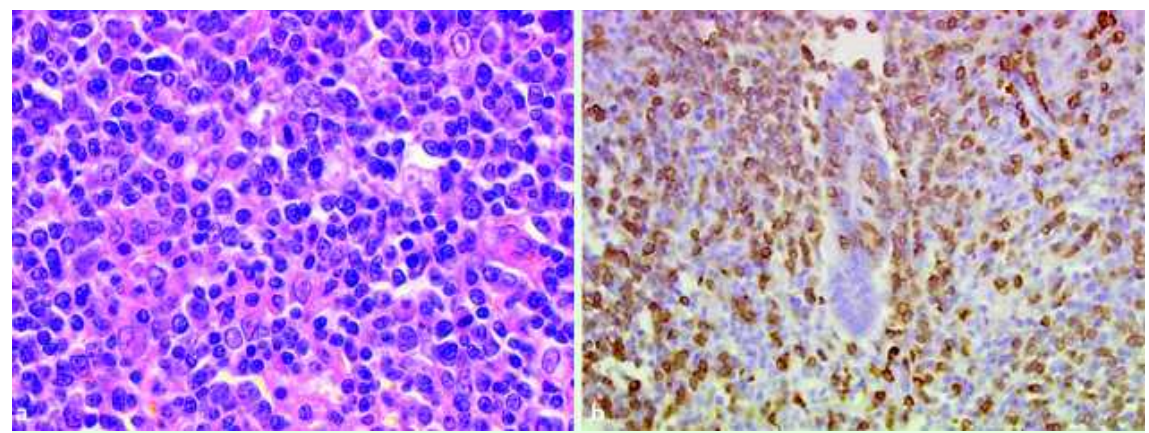

Fig. 3 a Microscopic features: small lymphoid cells, mainly centrocytic-like, rarely with plasma cell differentiation (hematoxylin-eosin-saffron stained section, magnification $\times 400$ ). b Immunostaining with anti-CD20: cells expressed CD20. Focal invasion of the crypt epithelium reminiscent of lymphoepithelial lesions (magnification $\times 100$ ).

the pseudotumoral presentation, defined as a tumoral presentation of LNH associated with inflammatory mesenteric lymph nodes [1,2]. The imperative differential diagnosis is low-grade lymphoma. In such cases, a diagnosis of malignancy is based on morphologic and immunophenotypic (histologic) features with molecular evidence of clonality [3].

LPH and LNH are similar, differing only in their endoscopic appearance. LNH appears as micronodules $1-2 \mathrm{~mm}$ in size, of a whitish-rose color, surrounded by normal mucosa [1]. In LPH, the micronodular endoscopic appearance is absent, giving a misleading picture of tumor [2] - as was observed in our cases. It is very important to take a large number of biopsies (sometimes macrobiopsies) to establish the diagnosis $[2,4]$. The physiopathology is not completely understood, but probably reflects an unspecific exacerbated lymphoid response to various stimuli (e.g., infections, allergies, immunodeficiency, autoimmunity) [1,2], which were identified in three-quarters of our patients.

There is no consensus about treatment, which is reserved for patients with severe symptoms. Treatments include surgery and hydrostatic barium enema (in the case of intestinal obstruction or intussusception), or the use of corticosteroids (with previous histologic confirmation of LPH) $[2,5]$. Due to its rarity, the natural history, and the increased risk of malignant transformation, many aspects such as clinical and endoscopic features and treatment follow-up remain uncertain.
Endoscopy_UCTN_Code_CCL_1AD_2AC

\section{J. Chaix ${ }^{1}$, C. Robles-Medranda ${ }^{2,3}$,}

S. Collardeau-Frachon ${ }^{4}$, H. Lukashok ${ }^{2}$, C. Le-Gall ${ }^{1}$, L. Michaud ${ }^{5}$, A. Lachaux ${ }^{1,3}$ Department of Pediatrics, Hepatogastroenterology Division, Hôpital Femme Mère Enfant - HFME, Groupement Hospitalier Est, Lyon, France Department of Hepatogastroenterology, Edouard Herriot Hospital, Lyon, France

CMR-Wilson, Edouard Herriot Hospital, Lyon, France

${ }^{4}$ Department of Pathology, Groupement Hospitalier Est, Lyon, France

Department of Pediatrics, Hepatogastroenterology Division, Jeanne de Flandre Hospital, Lille, France 
Table 1 Clinical, histologic, and endoscopic features of the patients.

\begin{tabular}{|c|c|c|c|c|c|c|c|c|c|c|}
\hline \multirow[t]{2}{*}{$\begin{array}{l}\text { Age } \\
\text { (y) }\end{array}$} & \multirow[t]{2}{*}{ Symptoms } & \multirow[t]{2}{*}{$\begin{array}{l}\text { Topo- } \\
\text { graphic } \\
\text { data }\end{array}$} & \multirow[t]{2}{*}{$\begin{array}{l}\text { Diagnostic } \\
\text { method }\end{array}$} & \multirow[t]{2}{*}{$\begin{array}{l}\text { Lesion } \\
\text { diameters }\end{array}$} & \multicolumn{2}{|c|}{$\begin{array}{l}\text { Immunohisto- } \\
\text { chemistry }\end{array}$} & \multirow[t]{2}{*}{ Etiology } & \multirow[t]{2}{*}{$\begin{array}{l}\text { Treat- } \\
\text { ment }\end{array}$} & \multirow[t]{2}{*}{ Response } & \multirow[t]{2}{*}{$\begin{array}{l}\text { Follow-up } \\
\text { (years) }\end{array}$} \\
\hline & & & & & Bcl-2 & CD20+ & & & & \\
\hline 4 & $\begin{array}{l}\text { diarrhea, } \\
\text { hemato- } \\
\text { chezia }\end{array}$ & $\begin{array}{l}\text { esophagus, } \\
\text { stomach, } \\
\text { duode- } \\
\text { num, } \\
\text { ileum, } \\
\text { colon }\end{array}$ & $\begin{array}{l}\text { gastros- } \\
\text { copy, } \\
\text { colonos- } \\
\text { copy }\end{array}$ & $20-30 \mathrm{~mm}$ & negative & negative & allergy? & - & $\begin{array}{l}\text { sponta- } \\
\text { neous Cl }\end{array}$ & 6 \\
\hline 7 & $\begin{array}{l}\text { intussus- } \\
\text { ception }\end{array}$ & $\begin{array}{l}\text { ileum, } \\
\text { colon }\end{array}$ & surgery & $15-20 \mathrm{~mm}$ & negative & positive & $\begin{array}{l}\text { HHV6 } \\
\text { virus }\end{array}$ & surgery & $\begin{array}{l}\text { good, no } \\
\text { recurrence }\end{array}$ & 2 \\
\hline 5 & $\begin{array}{l}\text { abdominal } \\
\text { pain }\end{array}$ & $\begin{array}{l}\text { jejunum, } \\
\text { ileum, } \\
\text { colon }\end{array}$ & $\begin{array}{l}\text { gastros- } \\
\text { copy, colo- } \\
\text { noscopy, } \\
\text { video } \\
\text { capsule }\end{array}$ & $7-15 \mathrm{~mm}$ & negative & positive & $\begin{array}{l}\text { immuno- } \\
\text { suppres- } \\
\text { sion }\end{array}$ & $\begin{array}{l}\text { cortico- } \\
\text { steroids }\end{array}$ & $\begin{array}{l}\text { good, LPH } \\
\text { improve- } \\
\text { ment, no } \\
\text { recurrence }\end{array}$ & 4 \\
\hline 12 & $\begin{array}{l}\text { rectal } \\
\text { bleeding }\end{array}$ & colon & $\begin{array}{l}\text { gastros- } \\
\text { copy, colo- } \\
\text { noscopy }\end{array}$ & $15 \mathrm{~mm}$ & negative & positive & $\begin{array}{l}\text { milk } \\
\text { allergy }\end{array}$ & diet & $\mathrm{Cl}$ & 0.6 \\
\hline
\end{tabular}

$\mathrm{y}=$ years, $\mathrm{mm}=$ millimetres, $\mathrm{Cl}=$ clinical improvement.

\section{References}

1 Rambaud JC, Galian A. Hyperplasie folliculaire lymphoide et lymphomes malins du tube digestif. In: Galmiche JP, Revillard JP, Teule-Espie M (eds). Immunité et tube digestif. Paris: John Libbey Eurotext, 1992: 95- 111

2 Vayre-Oundjian L, Boruchowicz A, Bloget F et al. Pseudotumor nodular lymphoid hyperplasia of the ileum. Gastroenterol Clin Biol 1997; 21: 990-993

3 Sukpanichnant S, Vnencak-Jones CL, McCurley $T L$. Determination of B-cell clonality in paraffin-embedded endoscopic biopsy specimens of abnormal lymphocytic infiltrates and gastrointestinal lymphoma by polymerase chain reaction. Am J Clin Pathol 1994; 102: 299-305
4 Shami VM, Waxman I. Lymphoid follicular proctitis mimicking rectal lymphoma: diagnosis by EMR. Gastrointest Endosc 2004 60: $648-652$

5 Shteyer E, Koplewitz BZ, Gross E et al. Medical treatment of recurrent intussusception associated with intestinal lymphoid hyperplasia. Pediatrics 2003; 111: $682-685$

Bibliography

DOI $10.1055 / \mathrm{s}-2008-1077668$

Endoscopy 2008; 40: E267-E268

(c) Georg Thieme Verlag KG Stuttgart · New York .

ISSN 0013-726X
Corresponding author

\section{J. Chaix, MD}

Hôpital Femme Mère Enfant, Service de Pédiatrie Générale, Gastroentérologie,

Hépatologie et Nutrition Pédiatriques

59 Boulevard Pinel

69677 Bron

France

Fax: +33-427857702

julie.chaix@chu-lyon.fr 